\title{
Evolution of Integrated Management Systems for Smart Library
}

\author{
Byung-Won Min, Yong-Sun Oh \\ Department of Information Communication Engineering \\ Mokwon University, Daejeon, 302-729, Korea
}

\begin{abstract}
For a library to be able provide information services and fulfill its function as a knowledge convergence center capable of responding to various information demands, the development of next-generation information systems based on the latest information and communication technology is needed. The development of mobile information services using portable devices such smart phones and tablet PCs and information systems which incorporate the concepts of cloud computing, SaaS (Software as a Service), annotation and Library2.0 is also required. This paper describes a library information system that utilizes collective intelligence and cloud computing. The information system developed for this study adopts the SaaS-based cloud computing service concept to cope with the shift in the mobile service paradigm in libraries and the explosion of electronic data. The strengths of such a conceptual model include the sharing of resources, support of multi-tenants, and the configuration and support of metadata. The user services are provided in the form of software on-demand. To test the performance of the developed system, the efficiency analysis and TTA certification test were conducted. The results of performance tests, It is encouraging that, at least up to 100MB, the job time is approximately linear and with only a moderate overhead of less than one second. The system also passed the level-3 or higher criteria in the certification test, which includes the SaaS maturity, performance and application program functions.
\end{abstract}

Keywords: Collective Intelligence, Cloud Computing, Smart Library Information System, SaaS, Metadata

\section{INTRODUCTION}

As The change in the information environment caused by the advance of information and communication technologies has occasioned the need for new changes in the contents, systems and services of libraries and data centers. The information system in the Web 2.0 environment emphasizes the participation, sharing and collaboration of users through Social Network Services (SNS) such as Facebook, Twitter, Flicker and blogs [1], [2]. Furthermore, the convenient mobility of information services using smart phones and tablet PCs is creating a ubiquitous environment in which information can be utilized anywhere and anytime for decision making and problem solving [3], [4].

To perform the function of a knowledge convergence center and thereby respond to a wide range of information demands, the development of mobile information services using portable devices such as smart phones and tablet PCs and information system which incorporate the concepts of cloud computing, SaaS, annotation and Library2.0 is required[5]-[8]. Furthermore, the collection of data produced by the relevant agencies and digitalization of the collected data are needed to develop the contents services to cope with the changing information environment [9]-[11].

\footnotetext{
* Corresponding author,Email: ysunoh@mokwon.ac.kr Manuscript received Oct. 29, 2012; revised Dec 07, 2012; accepted Dec 17, 2012
}

Many From a library perspective, as the advancement of computer and communication technology is making the Internet universally available, the ubiquitous environment, in which users can utilize contents anywhere and anytime while surfing on the sea of information, is realized. Users are using digital libraries with home computers or mobile devices over the wired and wireless networks. Such a ubiquitous environment also demands a new infrastructure to support the rapidly advancing terminals, networks and contents production technology [12].

Currently, most library systems use the client/server and ASP services for their software. However, such systems are difficult to manage and require high operating costs because of the problems related to high HW and SW purchasing costs, installation and distribution, customization, upgrade, fault and problem management, and expensive license royalties[13], [14].

Furthermore, library systems are not widely accepted by users as they are unable to create the information and knowledge needed by them. Although library systems provide useful information such as announcements, newly-arrived book lists, Q\&A, check-out status, recommended books, various research data, and new database data, such information is generally posted on the homepage as it is without filtering the information given by the information creators. As such, these systems are either difficult or limited for users.

Since the homepage provided to the users of a library system is centered on the library instead of the users, it is difficult even for users who are familiar with the library homepage to find the 
necessary information.

This paper proposes a technology for designing and deploying the smart library information system using collective intelligence and cloud computing to provide the needed information to the users by supplementing the problems described above.

This paper proposes a cloud-based integrated digital library system to overcome the problems of the existing digital library. It uses metadata to support customization for users. It differs from conventional methods in that it supports the tenants, which represent the user groups, with a software instance. It solves the weakness of ASP, which is not only costly to customize but also cannot take advantage of economy of scale because it loads each instance individually. Furthermore, there is very little initial investment since the library functions are standardized and modularized. They are designed and deployed as the cloud-based software on-demand type service model to enable easy, simple and low cost IT services.

\section{NEXT-GENERATION LIBRARY INFORMATION SERVICE UTILIZING COLLECTIVE INTELLIGENCE AND CLOUD COMPUTING}

\subsection{Definition}

The library information service of the future must go beyond just providing passive and simple knowledge accumulation and become a smart library that facilitates active knowledge creation and provides services through collective intelligence. Fig. 1 shows the basic concept of a smart library.

Most libraries nowadays create a wide range of information and provide it to users in addition to managing the information. Although libraries provide user training, announcements, etc. through their homepages, the information is often not delivered to users. However, some libraries recently adopted Web 2.0 to provide information, and that helped to properly deliver information to users. Therefore, which information to create and provide to the users has become a key issue for libraries. If libraries only continue providing information on new arrivals, announcements and databases, they may not gain the attention of users.

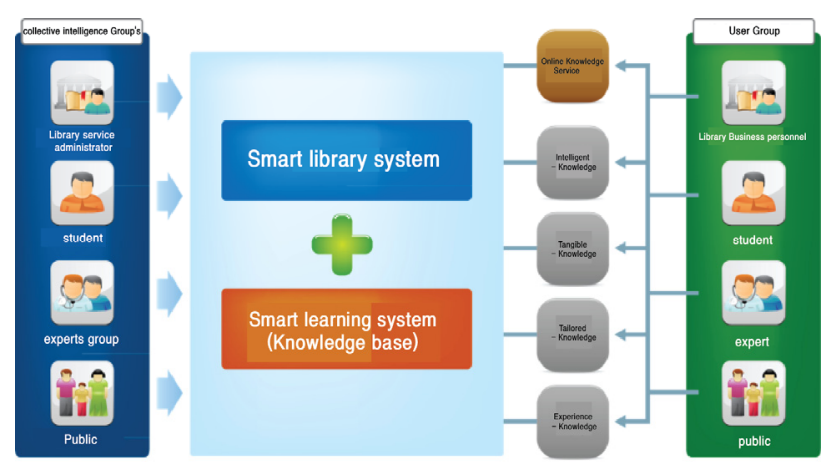

Fig. 1. Overview of Smart Library

Therefore, the future library information service must be a smart library which combines the existing library system functions with the knowledge-based e-learning system to develop creative human resources. It must be a system that enables the participants to develop their collective intelligencebased learning knowledge base using a collaborative and interactive interface, and which promotes self-managed learning to improve the creativity and logical thinking of the student.

While the existing library information service emphasizes the 1:n online knowledge service provided by library personnel, the smart library system can create, validate and categorize knowledge using collective intelligence group and provide intelligent knowledge, realistic knowledge, customized knowledge, and hands-on knowledge.

Furthermore, it enables contents sharing and interchanges of opinions using collective intelligence. The learning contents and knowledge base developed by collective intelligence can improve the national knowledge resource competitiveness, and smart tutoring in the next-generation e-learning environment can help achieve the national policy goals of developing creative human resources, improving public education, reducing private educational expenses, achieving a more balanced distribution of learning opportunities, and overcoming the gap between regions and social classes.

The smart library can play a central role in improving the country's position as a knowledge powerhouse in the era of the knowledge-based society by realizing nationwide lifelong learning and raising the educational level of the general public. The application of ubiquitous technology will enable the building of student-centered learning spaces and the restructuring of classrooms, and promote collaboration and cooperation between government agencies on the construction of a ubiquitous-based lifelong learning system.

\subsection{Smart Library information service system}

The main reason why users do not actively respond to the information provided by libraries is that the latter have failed to create the information and knowledge needed by them. Although the library system provides useful information such as announcements, newly-arrived book lists, Q\&A, check-out status, recommended books, various research data, and new database data, such information is generally posted on the homepage as is without filtering the information given by the information creators. As such, it is difficult to use or somewhat limited as far as users are concerned.

The smart library system of the future must allow the collective intelligence group to reprocess information so that users can easily understand and solve such problems. New tools designed for the efficient delivery of library knowledge and information must also be developed. Fig.2 below is a schematic diagram of a smart library service.

The library information service of the future must be developed with a new system so as to feature useful information/knowledge and to deliver it efficiently. If libraries do not create information and knowledge that promote library use, there will be no change in the user's perception of the library. Libraries must now extend the role of the service at the information management level to that of a knowledge base convergence repository to create and manage information and knowledge. Information and knowledge must go beyond being 
only of a guidance nature to stimulate users' desire to learn and to promote interest in libraries' data.

As shown in Fig.2, a smart library information service system provides the service converged with the intelligent tutoring system, as well as adding an intelligent learning engine, collective intelligence interactive interface, knowledge-base manager, and open framework technology to the conventional library information service system.

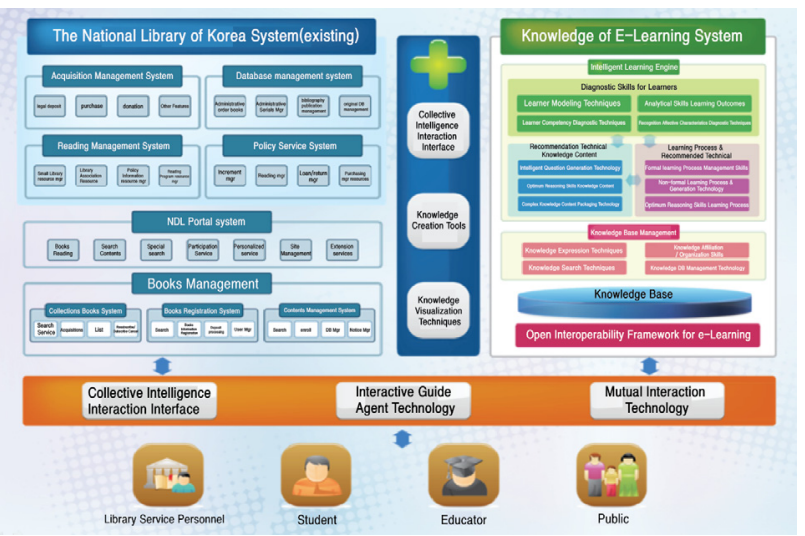

Fig. 2. Smart Library System Service Schematic Diagram

An 'intelligent learning engine' is an engine that improves students' creativity and logical thinking by providing them with the optimum learning process and knowledge contents intelligently, supporting the repetitive/dynamic learning process, and diagnosing their cognitive affective nature, thereby enabling them to engage in self-managed, explorative learning.

A 'knowledge base manager' is a management tool designed to systematically manage collective intelligence knowledge and to accurately and quickly search such knowledge in order to provide a self-managed and explorative learning environment.

A 'collective intelligence interactive interface' is an interface that supports experts' efforts to create knowledge content, provides various visualizations of the created knowledge, and supports interactive interaction between the users and the system.

An 'open e-learning interoperable framework' is a framework that provides a platform for e-learning interoperability and integrates legacy services.

An 'intelligent collective intelligence interactive learning agent' promotes learning and teaching to improve creativity and logical thinking by providing an interactive interface that promotes cooperation among the participants, a tutoring operation model for collective intelligence and cooperative learning, and augmented reality, virtual reality and explorative learning pattern analysis.

The 'learning platform and teaching/learning strategy model that supports the intelligent knowledge and composite/ dynamic/adaptive learning model' provides a learning platform through which students can receive the optimum education by dynamically restructuring and delivering the learning model such as the learning contents, progress and instructor according to the student's level, achievements, and inclination, as well as the level of difficulty of the contents.

Therefore, a smart library system is a next-generation, integrated learning system designed to cultivate and develop creative human resources. It is an intelligent tutoring system that uses a cooperative interactive interface to build a collective intelligence-based learning knowledge base and induce/support students' self-managed learning in order to improve their creativity and logical thinking.

\section{DEVELOPMENT OF THE SMART LIBRARY INFORMATION SERVICE SYSTEM}

\subsection{LinkSaaS Application Platform}

LinkSaaS Application Platform is software infrastructure designed to tame the data deluge with cost-effective, supercomputer-like capacity to process, transform, and indeed channel it for our benefit. It does this by leveraging the spare capacity of computing resources on the "edge" of the Internet resources which generally use only a small fraction of their capacity even when busy and are available for marginal operating cost and environmental impact - and allowing users and developers to combine and coordinate them in novel, dynamic ways. In order to assure the safety of these edge resources, LinkSaaS Application Platform builds upon Java applets, the best established security context for running untrusted, third-party code on the Internet; the use of this technology also seamlessly extends the reach of LinkSaaS Application Platform across different platforms, operating systems, and even enterprises. In principle, the LinkSaaS Application Platform network could encompass all the world's network-attached computers, providing an aggregate capacity 100 times greater than the 500 most powerful supercomputers combined.

The purpose of the LinkSaaS architecture is twofold: first, LinkSaaS is designed to increase the productivity of the installed base of computing devices. As noted above, most of the computers in the world use very little of their computing capacity even when "busy", e.g., editing a letter or spreadsheet, or browsing the Web. LinkSaaS aims to harness this spare capacity for general computing. However, there are significant technical obstacles to realizing this purpose. For this reason, LinkSaaS is designed to be simple to join, leave, and administer, with the resource owners remaining firmly in control of their computers; moreover, it must satisfy reasonable security expectations for running untrusted, third-party code.

Second, LinkSaaS is intended to increase the productivity of developers creating new applications for this large network. Distributed programming historically has been problematic and fraught with tedious details - in short, not usually worth the effort except in extreme cases. The easiest situation is where the developer only has a single computer cluster under his influence, but this cluster may not be large enough. Once administrative boundaries are crossed, even within the same organization, the developer must now keep track of a variety of configurations, accounts, and security mechanisms. In addition, the code usually has to be built and installed on platforms which all differ, even if only slightly (hence the tedious details). The code then has to be activated, and the network configuration itself, such as the presence of firewalls, must be considered. 
LinkSaaS addresses the above problem by specifying a streamlined environment for programming and deploying code across the network based on Java applets. This environment will be described in greater detail below. With LinkSaaS, developing, deploying, and provisioning a large-scale network application becomes realistic and practical.

\subsubsection{Description}

In this section, the LinkSaaS architecture is described. Its components are described first, followed by descriptions of how developers, users, and administrators see the resulting system. Every attempt has been made to keep LinkSaaS simple and straightforward to join, leave, program, and administer.

The LinkSaaS network itself provides a basic infrastructure which makes the network programmable much like a peer-topeer network while still respecting administrative constraints. It is not intended to make a distributed system look like a single computer; the authors suggest that this level of abstraction is best approached through software built on the LinkSaaS infrastructure. Moreover, the single computer abstraction is neither necessary nor appropriate in many circumstances; instead, programmers should be free to use the network creatively, and to construct network configurations appropriate to the nature and scale of the problems they are tackling.

To the extent that the LinkSaaS architecture is based on services which can be combined to form other services, it will be seen to bear some relation to a service-oriented architecture (SOA). However, LinkSaaS services do not have to reside on big, expensive servers; instead, they are provisioned by resources on the edge of the network. Also, unlike a typical SOA, LinkSaaS specifies a streamlined remote service deployment model, facilitating dynamic, highly distributed service provision; it thus relieves the programmer of some of the most tedious aspects of practical distributed programming.

Furthermore, LinkSaaS relies on the Java Virtual Machine (JVM), with its built-in security mechanisms, to accomplish remote deployments safely; it should be noted that Java applets have been running untrusted, third-party code in web browsers for over ten years. The JVM allows compiled code to run under Windows, Linux, Mac OS, and a number of other, less familiar operating systems, as well as on hardware ranging from the typical x86-based PC to an ARM-based mobile phone. The speed penalty for running code in the JVM has also largely vanished due to HotSpot runtime compilation into native machine code. By building on Java technology, LinkSaaS can offer secure, high-performance, cross-platform computing.

\subsubsection{Components}

Fig.3 shows a block diagram of a LinkSaaS network. The LinkSaaS network is divided into administrative domains, each of which can have one or more LinkSaaS servers. Each server is in turn connected to the Internet, through which it communicates with other servers as well as generic web servers. LinkSaaS clients then connect to one or more LinkSaaS servers; in principle, they could connect to servers in different domains, if their network access allows it.

It can be seen that the LinkSaaS network is an overlay network built, firstly, on the Internet itself. LinkSaaS servers are identified publicly by their Internet addresses, and since LinkSaaS servers and domains are fundamentally independent of one another, the LinkSaaS network is scalable in the same sense that the Internet itself is scalable. The network within a LinkSaaS domain may be different, however, and the clients may or may not have direct access to the Internet - for instance, because of a firewall, or its implementation on a proprietary network. Even so, the LinkSaaS clients will remain accessible to LinkSaaS network operations.

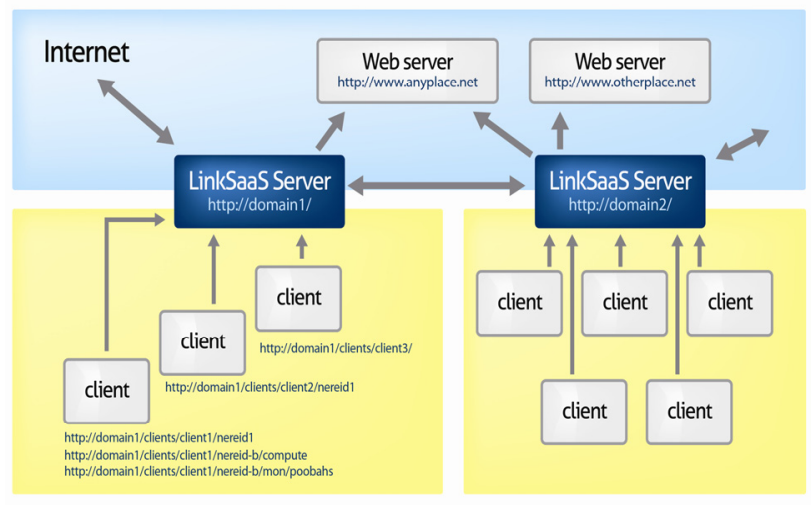

Fig. 3. Block diagram of the LinkSaaS network, showing servers, clients, and application/web servers.

\subsubsection{LinkSaaS Server}

The LinkSaaS server is the lynchpin of the LinkSaaS network: clients join the LinkSaaS network by connecting to a server, and the server acts as a proxy for the clients in communication with the wider network as well as with other clients (Fig.4).

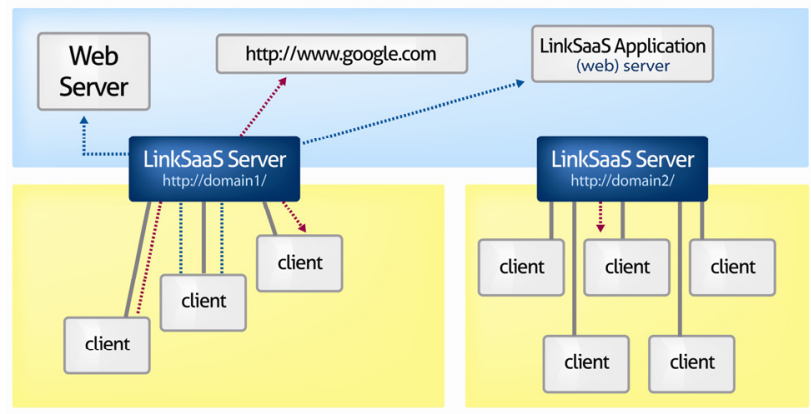

Fig. 4. Examples of communications in a LinkSaaS network, showing the central role of the LinkSaaS servers

In this way, the clients can communicate with the wider network even if they are protected behind a firewall - only the server need be exposed to the wider world. Within an administrative domain, a LinkSaaS server should be maintained and monitored as any central service, such as an e-mail service or an institutional web server. In turn, because of its central role in the LinkSaaS network, the server can act as an administrative control point for its clients, monitoring LinkSaaS bandwidth usage and limiting it if necessary.

Thus, even though clients can communicate with one another 
as if they were participating in a peer-to-peer network, the administrator can maintain a useful degree of control over their resource usage. The LinkSaaS architecture therefore addresses the common shortcomings in peer-to-peer network operation.

The LinkSaaS server manages the namespace of its domain, or, in other words, it assigns names to the clients connected to it. These names are globally unique by construction as long as LinkSaaS servers maintain unique IP addresses - which is an assumption of the Internet itself.

\subsubsection{LinkSaaS Client}

LinkSaaS clients are the workhorses of the LinkSaaS network: they are the programmable elements out of which developers create applications. The client itself is a program which runs on a host computer: it can be started from a web browser (applet client), run as a user program (stand-alone client), or installed as a persistent service (enterprise client). It is possible to have several clients running on a single computer; each client will have a unique identifier with which it identifies itself to the LinkSaaS server.

As noted above, a LinkSaaS client downloads its first service descriptor from a LinkSaaS server. This service descriptor tells the LinkSaaS client what to install in its root container. In the case of a stand-alone or enterprise client, the client can connect to more than one server simultaneously, initializing a root container with a different service descriptor for each - since each LinkSaaS server, potentially in different administrative domains, may implement different domain policies. It is the responsibility of the LinkSaaS server to decide whether or not to accept a connection from a particular LinkSaaS client.

The security context of an applet client is shown in Fig.5. In this case, the entire LinkSaaS client is encapsulated within a Java applet and its Security Manager.

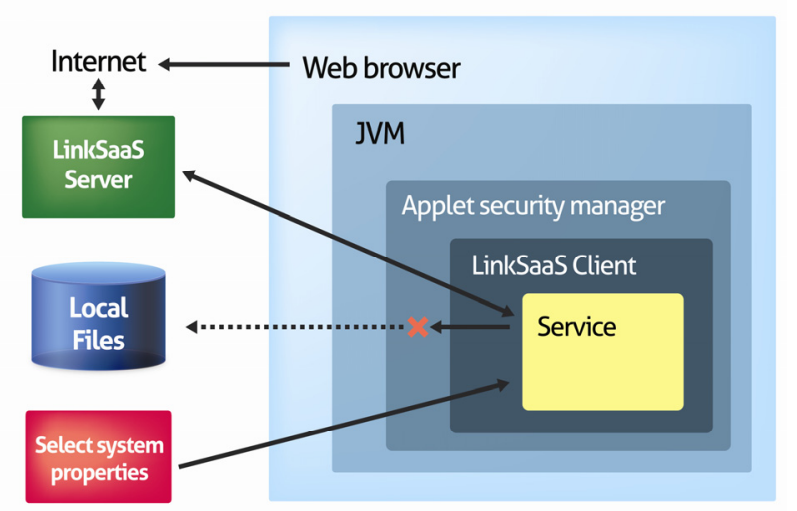

Fig. 5. LinkSaaS applet client security context. The service is prevented from reading local files or making arbitrary Internet connection

\subsubsection{LinkSaaS Application Server}

LinkSaaS clients are able to access normal web resources via the LinkSaaS server. This feature allows them to access not only the whole World Wide Web for information or other functionality, but it also allows the developer a convenient method to control his application.
As noted above, LinkSaaS does not specify a particular application model, but a particularly simple model, used already for several applications, consists of a web server (the application server) which provides a work queue and a user interface through which a user submits to it. Clients are set up to poll the application server with simple HTTP GET requests for work to do. Results can be sent back via HTTP POST operations or stored until fetched and cleared by the application server.

The application server is not a part of the LinkSaaS network per se, and indeed can be written without any reference to LinkSaaS code; its interaction with LinkSaaS clients can be managed entirely through normal web and socket connections made through the LinkSaaS server. The server therefore could be implemented using simple CGI scripts on an existing web server, though more flexibility may be obtained by using streamlined web server infrastructure software such as Grizzly or LinkSaaS's own NIONetworkHandler class. It should also be noted that a LinkSaaS service could also function as the application server.

\subsubsection{LinkSaaS Service}

A LinkSaaS service is a program that can be installed and run on a LinkSaaS client. Services can perform data processing, serve web content, and communicate with other services or external web sites. In addition, a LinkSaaS service can create child containers into which other LinkSaaS services can be installed.

It is worth noting the terminology: a LinkSaaS service runs on a LinkSaaS client, not a server. However, it can be said that a LinkSaaS server (which after all provides the root of the service's public URL) is what offers the service - even though the actual execution of the service is on another computer.

A LinkSaaS service is initialized by pointing the client to a web-accessible service descriptor in the form of an NML document. This descriptor can be located on any web server, not necessarily the application server or any LinkSaaS server (though the NML document describing the root container resides on the LinkSaaS server by design).

The LinkSaaS Markup Language (NML) has an XML-like syntax, with three tags defined:

- <LinkSaaS-app $>$ defines the application's name and encompasses all the classloaders and services it uses.

- <classloader $>$ defines the location of class hierarchies and JAR files for services defined within its scope.

- <service> defines an actual service with a name and a main class. Data between the opening and closing tags are passed to the service's initialization method as a character sequence (CharSequence).

The <classloader $>$ tag is optional; any services defined outside a <classloader $>$ scope is assumed to find classes starting from the service descriptor's URL directory. It is often convenient for any necessary class or JAR files to reside alongside the

service descriptor, but the <classloader> tag allows applications to draw upon classes from a variety of sources.

An example of an NML document describing a 
CommandService, an often-used root container, is shown below.

In this example, the application is called "command", and its Java classfile is found relative to the server's default web directory. The data between the $<$ service $>$ tags (in this case a primitive authorization string) is passed to the service on initialization.

An NML document can describe multiple services with different code sources. Services may be combined into larger services by including references in the initialization data, whether to other service names within the same NML document, or using URL's of external services. An "autoconfiguration" LinkSaaS service may be employed to generate further service descriptions as needed.

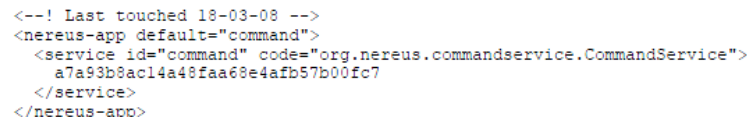

Fig. 6. NML document for CommandService descriptor

\subsubsection{LinkSaaS Api}

The API of a LinkSaaS service is much like that of a Java applet: if it is deployed in a standard JRE, then it can use any of that JRE's libraries. However, the usual applet security restrictions apply: it cannot modify system properties, cannot access the local filesystem directly, and cannot open network connections except to the server from which the applet was originally downloaded. Actions beyond these must be authorized by the client (and by implication, the user) through Java's extension mechanism.

The most basic LinkSaaS service interface (LinkSaaS Service) specifies only an init() entrypoint, which is called when the service is created. The name of the service is determined by the service descriptor; the client delegates URL's with this path element to the service.

An AbstractService class has been provided to make it easier to write new LinkSaaS services. AbstractService provides two abstract entrypoints, prepareToListen() and handleConnection(). prepareToListen() is invoked when the service is created (it is invoked by the AbstractService.init() method). handleConnection(), on the other hand, is called whenever a connection is made to the service from the outside, including the times when a user clicks into the service through the LinkSaaS server.

Because of the LinkSaaS client's applet-like constraints, several methods have been provided in AbstractService to facilitate communication with the outside world. First, println() and printErr() methods have been provided to print messages to the output and error panels in the LinkSaaS client's user interface. Web access must be redirected through the LinkSaaS server, which serves as a web proxy for its clients: a createRedirectedURI() method is provided to create the proxy URL from an original URL. A socket proxy is also provided.

The common application pattern described above is implemented on the service side by using prepareToListen() to create a new thread which periodically polls the application server (whose web address has been transformed by
createRedirectedURI()) for work to do. In this case, handleConnection() can be used by the application server to fetch stored results, or by other LinkSaaS services to fetch intermediate results. It can also respond to requests for status information, for instance by users clicking through the LinkSaaS server web page.

\subsubsection{LinkSaaS network and namespace}

One of the key benefits of the LinkSaaS network to network programming is to define a straightforward namespace by which elements of the network can be addressed. The LinkSaaS namespace consists of URL's, all of which are valid for public web access.

Each LinkSaaS server has a public URL such as http://domain1/, where "domain1" refers to the LinkSaaS server host. Since a LinkSaaS domain will typically have one or a few LinkSaaS servers, and policies are determined on a domain level and implemented by the servers, it is appropriate (though not necessary) to designate LinkSaaS servers with domain names. Since the LinkSaaS servers are publicly accessible Internet nodes, their names must be registered in the normal way.

The LinkSaaS server provides several web pages, such as http://domain1/clients, which lists the clients connected to it.

When clients connect to a LinkSaaS server, it creates a root container into which the default service, described by DomainRoot.nml, is installed. This service is addressed by appending the client alias to the clients subdirectory of the server URL, e.g., http://domain1/clients/client5. The client then delegates the interpretation of any further path elements to the service. Thus, if the service has created child containers in which it has installed services, those services are referred to in further path elements.

In principle, the entire LinkSaaS namespace is accessible via the Web. If a service needs to address another service, it creates a redirected URL out of the other service's public URL, just as if it was a normal web resource.

\subsubsection{LinkSaaS Application}

Software delivered as a service offers distinct advantages over software delivered by more traditional means: it is frequently mobile, web-based, centrally managed, and nearly free of troublesome installations and patches. In its simplest form, the architecture implied by these features consists of many clients attached to a large server infrastructure. Increasing numbers of users as well as improved functionality generally impact the server load and therefore drive server requirements, with increasingly expensive outcomes. Reducing server load therefore decreases the overall cost of the system, but risks substantially increasing operational costs. 


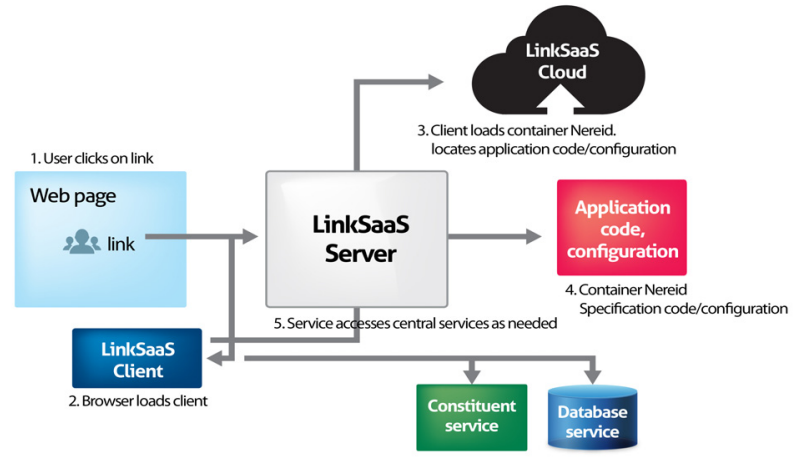

Fig. 7. Interactions within a LinkSaaS application

\subsection{Consideration}

To provide a user service that emphasizes Web service search, accessibility and usability, contents creation and recombination, interoperability and a personalized service, the smart library must be developed within the concept of Web 2.0 and Library 2.0.

To develop a smart library information service system, an open Web conforming to the $\mathrm{W} 3 \mathrm{C}$ standard is needed first of all.

- Web page supporting all browser versions of Netscape 7.x,

MS IE 6.x, and Firefox 1.x or higher (cross browsing supported)

- Various operating environments: Web accessibility and usability

- Web service environment with consideration to generality and scalability

- Standardization: Mutual interfaces of the Web service and integrated usage

- Administrative Agency Homepage Development/ Operation Standard Guidelines"

•"W3C (Worldwide Web Consortium) Web Standard Technology Recommendations"

- Web service standard architecture: XML, UDDI, WSDL, and REST

- Information System Development/Operation Technical Guidelines 2.0"

Secondly, various mash-up services using open API (Application Programming Interface) must be considered. Lastly, user authentication and access privilege management must be provided using the homepage login linking (SSO).

\section{ANALYSIS OF THE PERFORMANCE OF THE PROPOSED SYSTEM}

\subsection{Test Items}

In this As shown in Table 1, the various items for the TTA certification test of the proposed smart library information service system comprise four items in SaaS maturity, three items in performance, and thirteen items in application program function.

The SaaS maturity test items include multi-tenant support, same code support, customizing support, and data sharing support. The performance test items include the CPU utilization rate, memory utilization rate, and response time. The application program performance test items include the received book system, catalog system, inspection system, academic journal table of contents system, periodicals, original document copying service, system management, DL contents management, research report management, contents management, original copy management service, ETM service, and general user service.

Table 1. Test Items

\begin{tabular}{|c|c|}
\hline Item & Details \\
\hline SaaS Maturity & $\begin{array}{l}\text {-. Multi-tenant support, Same code support } \\
\text {-. Customizing support, Data sharing support }\end{array}$ \\
\hline Performance & $\begin{array}{l}\text {-. CPU utilization rate, Memory utilization rate } \\
\text {-. Response time }\end{array}$ \\
\hline $\begin{array}{c}\text { Application } \\
\text { Program Function }\end{array}$ & $\begin{array}{l}\text {-. Received book system, Catalog system } \\
\text {-. Inspection system, Periodicals } \\
\text {-. Academic journal table of contents system } \\
\text {-. Original document copying service } \\
\text {-. System management } \\
\text {-. DL contents management, } \\
\text {-. Research report management } \\
\text {-. Contents management, General user service } \\
\text {-. Original copy management service }\end{array}$ \\
\hline
\end{tabular}

\subsection{Test Result}

Table 2. shows the results of the TTA certification test. It indicates that each item of the cloud-based smart library information service system operates normally.

Table 2. Test Result

\begin{tabular}{|l|c|c|}
\hline \multicolumn{1}{|c|}{ Performance Test Item } & Unit & Measurement \\
\hline 1. SaaS Maturity Model & Level & Level 3 \\
\hline (1) Connection & Instance & N (customer): 1 (instance) \\
\hline (2) Provided program & Code & Same code \\
\hline (3) Customizing & Configuration & $\begin{array}{c}\text { Configurable by the } \\
\text { customer for each tenant }\end{array}$ \\
\hline (4) Economy of scale & Yes/No & Yes (instance sharing) \\
\hline (5) Scaling & Yes/no & $\begin{array}{c}\text { Replacement to better } \\
\text { performing system }\end{array}$ \\
\hline (6) Data & Format & Data sharing \\
\hline 2. Performance Test & Class & Class A \\
\hline (1) CPU utilization rate & $\%$ & $8 \%$ or less \\
\hline (2) Memory usage & Byte & $500 \mathrm{MB}$ or less \\
\hline (3) Response time & Sec & 1 Sec \\
\hline (4) Portability & Yes/No & Yes \\
\hline
\end{tabular}

The results of performance tests, from searching text without the benefit of an index, are shown in Fig 8. It is encouraging that, at least up to $100 \mathrm{MB}$, the job time is approximately linear and with only a moderate overhead of less than one second. 


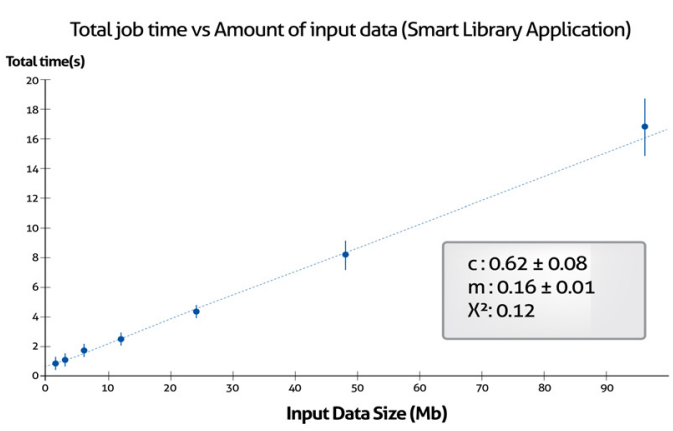

Fig. 8. Job performance of Smart Library application

\section{CONCLUDING REMARKS}

The greatest problem concerning library homepages up until now has been that they are not centered on the users but on the library, which is the information provider. As a result, it is not easy even for users familiar with the library homepage to find the desired information on the homepage, which suggests that the use of the various electronic data provided by a library homepage is not that high, particularly because the users still perceive books as the main source of information provided by libraries.

To solve this problem, this paper emphasizes that the new Web 2.0 based library service needs to break away from simply acting as an information manager and play the role of information and knowledge producer. To that end, this paper presents the smart library information service system as a means for libraries to produce and provide information and knowledge centered on the users through its library homepage.

Currently, most library systems use client/server and ASP services for their software. However, such systems are difficult to manage and incur high operating costs because of the problems related to high HW and SW purchasing costs, installation and distribution, customization, upgrade, fault and problem management, and expensive license royalties.

To solve such problems, this study developed the key elements for deploying the cloud-based digital library system in the multi-tenant environment. The system was deployed with the SaaS-based software on-demand type service model, which requires only a small initial investment, is simple and easy to use, and delivers a low cost IT service.

\section{REFERENCES}

[1] H.S. Shin, "Understanding MS Cloud Computing and the Edger Service Platform”, ZDNet Korea, 2008(12).

[2] Y.M. Kim, “The Web-Based SaaS Platform”, 2008. 12. 17,http://www.software.or.kr/ICSfile/afieldfile/2008/12/1 8/5.pdf

[3] Digital Times, "SaaS and the Future of Software", 2006. 05.04.

[4] S.H. Han, Study of Thesaurus Updating Using Collective Intelligence, Journal of the Korea Society for Information Management, vol. 26, no. 4, 2009(9), pp.25-43.
[5] http://www.naver.com

[6] S.J. Kwak, "Study of the National Library Operation Plan", The National Library of Korea, 2011(3).

[7] B.H. Yang, Web 2.0-Based Library information service, Information Management Studies, vol.39, no.1, 2008, pp.199-220.

[8] Sasan Adibi, "Data Mining - A Captured Wired Traffic Approach", IJAST volume 21, August 2010, pp.11-30.

[9] Ploypailin Intapong, Sittapong Settapat, Boonserm Kaewkamnerdpong and Tiranee Achalakul, "Modular Web-Based Collaboration Platform", IJAST volume 22, September 2010, pp.37-48.

[10] Dinesh C. S. Bisht, Ashok Jangid, "Discharge Modelling using Adaptive Neuro - Fuzzy Inference System", IJAST Volume 31, June 2011, pp.99-114.

[11] B.W. Min and Y.S. Oh, Design of the SaaS PlatformBased Integrated Digital Library System, Proceedings of the Korea Contents Association 2010 Spring Symposium, 2010, pp.447-449.

[12] B.W. Min, H.Y. Oh, and Y.S. Oh, Improvement of the SaaS-Based Digital Library Integrated Management Service System, Proceedings of the KOCON 2011 Spring Integrated International Digital Design Exhibition, 2011, pp.3-4.

[13] B.W. Min and Y.S. Oh, Implement of Integrated Management System for Digital Library Supporting Multitenent Environment Based on SaaS, Journal of the Korea Contents Association, pp.93-103, 2011, vol. 11, no. 5.

[14] H.Y. Oh, B.W. Min and Y.S. Oh, "User Customized Web Interface Design optimized for SaaS-based Digital Library System", Journal of the Korea Contents Association, vol. 11, no. 5, 2011, pp.148-156.

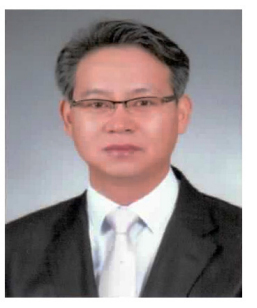

\section{Byung-Won Min}

$\mathrm{He} \mathrm{He}$ received M.S degree in computer software from Chungang University, Seoul, Korea in 2005. He worked as a professor in the department of computer engineering, Youngdong University, Youngdong, Chungbuk, Korea, from 2005 to 2008.

$\mathrm{He}$ received $\mathrm{Ph} . \mathrm{D}$. degrees in Information and Communication Engineering from Mokwon University, Daejeon, Korea., in 2010, respectively. His research interests include digital communication systems, information theory and their applications.

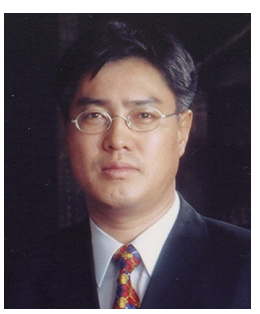

\section{Yong-Sun Oh}

$\mathrm{He}$ received B.S., M.S., and Ph.D. degrees in electronic engineering from Yonsei University, Seoul, Korea, in 1983, 1985, and 1992, respectively.

He worked as an R\&D engineer at the System Development Division of Samsung Electronic Co. Ltd., Kiheung, Kyungki-Do, Korea, from 1984 to 1986 . He joined the faculty of the Dept. of Information Communication Engineering, Mokwon University, Daejeon, Korea, in 1988. During 1998- 
1999 he served as a visiting professor of Korea Maritime University, Busan, Korea, where he was nominated the head of the Academic Committee of the Korea Institute of Maritime Information and Communication Sciences. Afterward, he returned to Mokwon University in 1999 and served as Dean of the Central Library and Information Center from 2000 to 2002. and as Director of the Corporation of Industrial Educational Programs from 2003 to 2005 . Recently he worked as an Invited Researcher at ETRI(Electronics and Telecommunications Research Institute) from 2007 to 2008. He is currently a professor of Mokwon University and the President of Korea Contents Association.

His research interests include digital communication systems, information theory and their applications. Recently he is interested in multimedia content and personalized e-Learning. 